\title{
Physical modeling of Fermi-level effects for decanano device process simulations
}

I. Martin-Bragado, ${ }^{*}$ R. Pinacho, P. Castrillo, M. Jaraiz, J.E. Rubio, and J. Barbolla Department of Electronics, University of Valladolid. Campus M. Delibes, 47011 Valladolid, Spain

\begin{abstract}
We report on a physically-based Fermi-level modeling approach designed to be accurate and yet amenable to be implemented in a device-size process simulator. We use an atomistic kinetic Monte Carlo method in conjunction with a continuum treatment for carrier densities. The model includes (i) charge reactions and electric bias according to the local Fermi-level, (ii) pairing and break-up reactions involving charged particles, (iii) clustering-related dopant deactivation and (iv) Fermi level-dependent solubility. Degenerated statistics, band-gap narrowing, and damage-induced electrical compensation are also included. The parameters used for charged particles are in agreement with ab-initio calculations and experimental results. This modeling scheme has proved to be very computationally efficient for realistic device-dimension process simulations. We present an illustrative set of simulation results for two common dopants, Boron and Arsenic, and discuss the potential of this approach for accurate process simulation of decanano CMOS devices.
\end{abstract}

*Electronic address: ignacio.martin@tel.uva.es 


\section{INTRODUCTION}

Current decananometer CMOS technology faces complexities arising from the need for very high-doping, reduced thermal budged (non-equilibrium conditions), and 3-dimensional effects. Under such conditions it would be advantageous to be able to directly implement the underlying physical mechanisms. In particular, high-doping concentrations demand a correct description of extrinsic diffusion [1, 2]. Other charge-related issues are Fermi-level dependent solubility [3], clustering-related dopant deactivation [4], and high-damage electrical compensation.

The non-lattice kinetic Monte Carlo method (kMC) has proved to offer a detailed and comprehensive description of technology process physics together with the capability of simulating typical device sizes and processing times $[5,6]$. In this work we describe a physically-based Fermi-level modeling approach for atomistic kMC. This approach has been designed for its implementation in the process simulator DADOS [7, 8]. We will first focus on the physical models, then outline the implementation approach, and finally show some simulation examples.

\section{PHYSICAL MODELS}

In our simulation scheme, particles and defects are treated atomistically, while carrier concentrations are treated in a continuum fashion. Charge reactions are much faster than structural reactions [9] and, therefore, electrical equilibrium can be assumed even in situations in which particle concentrations are far from equilibrium conditions. Consequently, the Fermi-level $\left(\mathrm{e}_{\mathrm{F}}\right)$ can be considered to be well defined. In this section we will adapt the formulation of Fermi-level effects in diffusion [1] to an atomistic framework and will introduce some concepts not explicitly present in continuum model formalisms.

We are going to take the vacancy (V) as an example of a simple point defect. The silicon vacancy has multiple charge states: $\mathrm{V}^{0}, \mathrm{~V}^{-}, \mathrm{V}^{--}, \mathrm{V}^{+}, \mathrm{V}^{++}$. Its charge levels are well 
characterized $[1,10]$. We use the values listed in Table I. We denote by $e_{V^{-}}, e_{V^{--}}, e_{V^{+}}$, and $e_{V^{++}}$the energy levels associated to the charge transitions between $\mathrm{V}^{0}$ and $\mathrm{V}^{-}, \mathrm{V}^{-}$and $\mathrm{V}^{--}, \mathrm{V}^{0}$ and $\mathrm{V}^{+}$, and $\mathrm{V}^{+}$and $\mathrm{V}^{++}$, respectively. Then, the relative concentrations are:

$$
\begin{aligned}
& {\left[V^{-}\right] /\left[V^{0}\right]=\exp \left(\left(e_{F}-e_{V^{-}}\right) / k T\right)} \\
& {\left[V^{+}\right] /\left[V^{0}\right]=\exp \left(\left(e_{V^{+}}-e_{F}\right) / k T\right)} \\
& {\left[V^{--}\right] /\left[V^{0}\right]=\exp \left(\left(2 e_{F}-e_{V^{-}}-e_{V^{--}}\right) / k T\right)} \\
& {\left[V^{++}\right] /\left[V^{0}\right]=\exp \left(\left(e_{V^{+}}+e_{V^{++}}-2 e_{F}\right) / k T\right)}
\end{aligned}
$$

where $\mathrm{kT}$ is the thermal energy. We have assumed the same degeneracy factor for all charged states [1]. The concentration of $\mathrm{V}^{0}$ in equilibrium conditions (denoted as $\left[\mathrm{V}^{0}\right]^{*}$ ) is controlled by its formation energy, $\mathrm{E}_{\mathrm{f}}\left(\mathrm{V}^{0}\right)$, that is independent on $\mathrm{e}_{\mathrm{F}}$ because it is a neutral particle. The Fermi-level dependence of the formation energies of the charged states, as well as their equilibrium concentrations, can be obtained from Eqs.1. The dependence of the formation energies with $e_{F}$ for charged vacancies using the values of Table I are displayed in Fig.1. As a convention, we take the origin of energy levels at the valence-band edge. The total vacancy concentration as a function of $\mathrm{e}_{\mathrm{F}}$ will be primarily determined by the charge state with lower $\mathrm{E}_{\mathrm{f}}$ (thick dashed line in the Figure). As it can be seen in the Figure, $\mathrm{V}^{+}$is a metastable state, i.e. it is not the minimum $\mathrm{E}_{\mathrm{f}}$ for any value of $\mathrm{e}_{\mathrm{F}}$. Out from equilibrium, Eqs.1 are still valid and, therefore, the vacancy supersaturation, defined as $S_{V}=[\mathrm{V}] /[\mathrm{V}]^{*}$, is the same for all the charge states: $\left[\mathrm{V}^{\mathrm{j}}\right] /\left[\mathrm{V}^{\mathrm{j}}\right]^{*}=\left[\mathrm{V}^{0}\right] /\left[\mathrm{V}^{0}\right]^{*}=\mathrm{S}_{\mathrm{V}}$.

The migration frequency $v_{\mathrm{m}}$ of the vacancy in a given charge state $\mathrm{j}$ is:

$$
v_{m}\left(V^{j}\right)=v_{m 0}\left(V^{j}\right) \exp \left(-E_{m}\left(V^{j}\right) / k T\right)
$$

where $v_{\mathrm{m} 0}$ is the migration prefactor and $\mathrm{E}_{\mathrm{m}}$ the migration energy. The diffusivity of $\mathrm{V}^{\mathrm{j}}$ will be:

$$
D\left(V^{j}\right)=\lambda^{2} v_{m}\left(V^{j}\right) / 6
$$


where $\lambda$ is the jump distance. In equilibrium conditions, the product $D\left(V^{j}\right)\left[V^{j}\right]^{*}$ will be proportional to $\exp \left(E_{\mathrm{dif}}\left(\mathrm{V}^{\mathrm{j}}\right) / \mathrm{kT}\right)$, being $\mathrm{E}_{\mathrm{dif}}=\mathrm{E}_{\mathrm{m}}+\mathrm{E}_{\mathrm{f}}$ the diffusion energy, that is also indicated in Fig. 1. The diffusion flux will be dominated by the defect with lower $\mathrm{E}_{\text {dif }}$ (thick solid line in Fig. 1). Using the parameters of Table I a good fit to V diffusivity measurements of Ref.11 is achieved.

The charge levels of silicon self-interstitials are not conclusively established $[1,10,12] . I^{+}$ seems to be metastable but with a very low migration energy and probably controls interstitial diffusion for p-doped materials [10]. As a simplification, we will ignore the $\mathrm{I}^{++}$state [1] and will describe the interstitial using the $\mathrm{I}^{+}, \mathrm{I}^{0}$, $\mathrm{I}^{-}$states, using the values listed in table $\mathrm{I}$. These parameters are set to fit the experimental data of both I diffusion [13] and total silicon selfdiffusion [14].

Electric drift is a natural consequence of the local dependence of the formation energies of charged particles. Within the kMC framework, the relation between the migration frequency in the positive and negative directions along the $\mathrm{x}$ axis for a migrating point defect with charge jq is

$$
\frac{v_{m,+x}}{v_{m,-x}}=\exp \left(\frac{j q F_{x} \lambda}{k T}\right)=\exp \left(-\frac{j q \lambda}{k T} \frac{d e_{F}}{d x}\right)
$$

where $\mathbf{F}$ is the electric field and $q$ is the absolute value of the electron charge. Using Eq.4 in a first order approximation, the $\mathrm{x}$-component of drift velocity, $\mathrm{v}_{\mathrm{x}}=\lambda\left(\mathrm{v}_{\mathrm{m},+\mathrm{x}}-v_{\mathrm{m}, \mathrm{x}}\right)$, can be written as $\mathrm{v}_{\mathrm{x}}=(\mathrm{jqD} / \mathrm{kT}) \mathrm{F}_{\mathrm{x}}$, that is the well-known Einstein relation. Electric drift has to be accounted properly in order to reproduce the fact that, in equilibrium, $\left[\mathrm{V}^{0}\right]^{*}$ and $\left[\mathrm{I}^{0}\right]^{*}$ have to be constant even if $\nabla e_{F} \neq 0$.

Let us consider now the cases in which the point defect can be seen as the pair AX, where A is an impurity and $\mathrm{X}$ is either an $\mathrm{I}$ or a $\mathrm{V}$. We are going to illustrate it with the case of interstitial boron $\mathrm{B}_{\mathrm{i}}$ (that, in our non-lattice $\mathrm{kMC}$ description, is equivalent to a $\mathrm{BI}$ pair). It is commonly accepted that $\mathrm{B}$ migrates mainly due to interstitial mechanism, via $\mathrm{B}_{\mathrm{i}}$, rather than 
via V-mediated mechanism. The charged states of $\mathrm{B}_{\mathrm{i}}$ are $\mathrm{B}_{\mathrm{i}}{ }^{-}, \mathrm{B}_{\mathrm{i}}{ }^{0}$ and $\mathrm{B}_{\mathrm{i}}^{+}$. The pairing and break up and charge reactions related to $\mathrm{B}_{\mathrm{i}}$ can be represented by

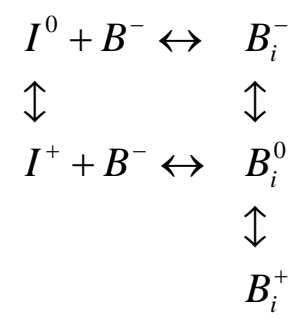

We assume substitutional boron to be immobile and always ionized (B`). In Eq.5 horizontal reactions (pairing and break-up) conserve the charge while vertical reactions establish the electrical equilibrium. Direct break-up of $\mathrm{B}_{\mathrm{i}}^{+}$is not included because we do not consider $\mathrm{I}^{++}$. The charge level values that we adopt for $\mathrm{B}_{\mathrm{i}}$ are listed in table $\mathrm{I}$. These values have been measured by electrical characterization [10].

From the first reaction in Eq.5, in local equilibrium conditions:

$$
\frac{\left[B_{i}^{-}\right]}{\left[B^{-}\right]}=v_{c a p t} \frac{v_{m}\left(I^{0}\right)\left[I^{0}\right]}{v_{b k}\left(B_{i}^{-}\right)}
$$

being $\mathrm{v}_{\text {capt }}$ the effective capture volume for pairing reaction and $v_{\mathrm{bk}}$ the break-up frequency. The ratio of Eq.6 is independent of $\mathrm{e}_{\mathrm{F}}$ because the charges of $\mathrm{B}_{\mathrm{i}}^{-}$and $\mathrm{B}^{-}$are the same. In contrast, $\left[\mathrm{B}_{\mathrm{i}}^{0}\right] /\left[\mathrm{B}^{-}\right]$and $\left[\mathrm{B}_{\mathrm{i}}^{+}\right] /\left[\mathrm{B}^{-}\right]$are Fermi-level dependent. The activation energy for $\mathrm{B}_{\mathrm{i}}^{-}$ break-up will be $E_{b}\left(B_{i}\right)+E_{m}\left(I^{0}\right)$, $E_{b}$ denoting the binding energy. Considering energy conservation in reactions of Eq.5, it can be obtained that

$$
E_{b}\left(B_{i}^{0}\right)=E_{b}\left(B_{i}^{-}\right)+e_{B_{i}^{-}}-e_{I^{+}}
$$

and the activation energy for $\mathrm{B}_{\mathrm{i}}{ }^{0}$ break-up will be $\mathrm{E}_{\mathrm{b}}\left(\mathrm{B}_{\mathrm{i}}{ }^{0}\right)+\mathrm{E}_{\mathrm{m}}\left(\mathrm{I}^{+}\right)$. From equilibrium conditions it can be also derived that the break-up prefactors $v_{\mathrm{bk} 0}$ have to fulfill:

$$
v_{b k 0}\left(B_{i}^{0}\right) / v_{b k 0}\left(B_{i}^{-}\right)=v_{m 0}\left(I^{+}\right) / v_{m 0}\left(I^{0}\right)
$$

In Fig. 2 we have represented the formation energy of a $B_{i}^{j}$ from a $B^{-}\left(E_{f}\left(B_{i}^{j}\right)-E_{f}\left(B^{-}\right)\right)$as a function of $e_{F}$ (dashed lines). This is also the activation energy of the ratio $\left[\mathrm{B}_{\mathrm{i}}^{\mathrm{j}}\right] /\left[\mathrm{B}^{-}\right]$. The Figure is a good agreement with the picture derived from ab-initio calculations [15]. 
Boron effective diffusivity $\mathrm{D}(\mathrm{B})$ is given by the sum of the contribution of all the mobile species:

$$
D(B) \approx D\left(B_{i}^{-}\right) \frac{\left[B_{i}^{-}\right]}{\left[B^{-}\right]}+D\left(B_{i}^{0}\right) \frac{\left[B_{i}^{0}\right]}{\left[B^{-}\right]}+D\left(B_{i}^{+}\right) \frac{\left[B_{i}^{+}\right]}{\left[B^{-}\right]}
$$

Using the Maxwell-Boltzmann approximation, this equation is usually written in continuum models as:

$$
D(B)=D_{X}(B)+D_{P}(B) \frac{p}{n_{i}}+D_{P P}(B)\left(\frac{p}{n_{i}}\right)^{2}
$$

$\mathrm{p}$ and $\mathrm{n}_{\mathrm{i}}$ being the hole concentration and the intrinsic concentration, respectively. The relations between the above diffusivity components $D_{X}(B), D_{P}(B), D_{P P}(B)$ and the microscopic parameters are, therefore:

$$
\begin{aligned}
& D_{X}(B)=v_{\text {capt }} D\left(I^{0}\right)\left[I^{0}\right]\left(v_{m}\left(B_{i}^{-}\right) / v_{b k}\left(B_{i}^{-}\right)\right) \\
& D_{P}(B)=v_{\text {capt }} D\left(I^{0}\right)\left[I^{0}\right]\left(v_{m}\left(B_{i}^{0}\right) / v_{b k}\left(B_{i}^{-}\right)\right) \exp \left(\left(e_{B_{i}^{-}}-e_{i}\right) / k T\right) \\
& D_{P P}(B)=v_{\text {capt }} D\left(I^{0}\right)\left[I^{0}\right]\left(v_{m}\left(B_{i}^{+}\right) / v_{b k}\left(B_{i}^{-}\right)\right) \exp \left(\left(e_{B_{i}^{-}}+e_{B_{i}^{+}}-2 e_{i}\right) / k T\right)
\end{aligned}
$$

$\mathrm{e}_{\mathrm{i}}$ being the intrinsic level. These expressions can be used as a bridge between the parameters of continuum simulators and those of a kMC simulator. The activation energies of the terms in Eq.9 (usually known as "defect-assisted diffusion energies " or just "diffusion energies", $\mathrm{E}_{\mathrm{dif}}\left(\mathrm{B}_{\mathrm{i}}^{\mathrm{j}}\right)$ ) are represented in Fig.2. It can be seen that $\mathrm{B}_{\mathrm{i}}^{0}$ is a metastable state (as $e_{B_{i}^{+}}>e_{B_{i}^{-}}$) but is the main responsible for boron diffusion in a wide range of $e_{F}$ and, in consequence, $\mathrm{D}(\mathrm{B}) \propto \mathrm{p} / \mathrm{n}_{\mathrm{i}}$ [2]. According to Fig.2 the effective migration energy of a $\mathrm{B}_{\mathrm{i}}$ for $\mathrm{e}_{\mathrm{F}} \approx \mathrm{e}_{\mathrm{i}}$ is $\mathrm{E}_{\mathrm{dif}}\left(\mathrm{B}_{\mathrm{i}}{ }^{0}\right)-\mathrm{E}_{\mathrm{f}}\left(\mathrm{B}_{\mathrm{i}}^{+}\right) \approx 0.6 \mathrm{eV}$, in agreement with the experimental observation [16]. The parameters listed in table I have been chosen to fit the Arrhenius plot of equilibrium boron diffusivity, $\mathrm{D}(\mathrm{B})^{*}$, reported in Ref.17 for intrinsic conditions.

A similar analysis can be done for arsenic, but taking into account both vacancy and interstitial contributions, related to $\mathrm{AsV}$ and $\mathrm{As}_{\mathrm{i}}$, respectively. Our parameter set for $\mathrm{As}$ is reported in Ref.18. 
The distance traveled by a point defect pair before breaking up (usually denoted as the "long hop distance", $\Lambda$ ) is given by [19, 20]:

$$
\Lambda=\lambda \sqrt{\frac{1}{6} \frac{v_{m}}{v_{b k}}}
$$

In the above mentioned case of a $\mathrm{B}_{\mathrm{i}}$ for which multiple charged states are considered, Eq.12 becomes:

$$
\Lambda=\lambda \sqrt{\frac{1}{6} \frac{v_{m}\left(B_{i}^{-}\right) \frac{\left[B_{i}^{-}\right]}{\left[B^{-}\right]}+v_{m}\left(B_{i}^{0}\right) \frac{\left[B_{i}^{0}\right]}{\left[B^{-}\right]}+v_{m}\left(B_{i}^{+}\right) \frac{\left[B_{i}^{+}\right]}{\left[B^{-}\right]}}{v_{\text {capt }}\left(v_{m}\left(I^{0}\right)\left[I^{0}\right]+v_{m}\left(I^{+}\right)\left[I^{+}\right]\right)}}
$$

The temperature dependence of $\Lambda$ using the parameters of table I is displayed in Fig.3, both for intrinsic and doped silicon, and compared with experimental values for almost intrinsic materials [19, 21]. With the values we are using, $\Lambda \propto \mathrm{p} /$ ni for $\mathrm{e}_{\mathrm{F}} \sim$ ei.

The solubility of charged species depends on $\mathrm{e}_{\mathrm{F}}$ [3]. For the case of an acceptor, like boron, the solubility limit is given by:

$$
\left[B^{-}\right]_{\max }(T)=\left[B^{-}\right]_{\max }^{\mathrm{int}}(T) \exp \left(\frac{e_{F}-e_{i}}{k T}\right)
$$

where $\left[B^{-}\right]_{\max }^{\text {int }}=\left(\left[B^{-}\right]_{\max }^{e x t}\right)^{2} / n_{i}$ is the solubility in intrinsic conditions $\left(\mathrm{e}_{\mathrm{F}}=\mathrm{e}_{\mathrm{i}}\right)$ and $\left[B^{-}\right]_{\max }^{\text {ext }}$ the solubility when $\left[\mathrm{B}^{-}\right]_{\max } \gg \mathrm{n}_{\mathrm{i}}$. Equation 14 is a consequence of the fact that the formation energy of charged defects depends on $\mathrm{e}_{\mathrm{F}}$. Extended defects, such as clusters, are assumed to be electrically inactive and account for clustering related dopant deactivation.

\section{IMPLEMENTATION}

In atomistic kMC each individual defect is represented by one or more particles. Physical mechanisms are described as "interactions" (reactions between particles within a capture radius, for example: pairing) or as "events" (performed by the defects with a certain frequency, for example: break-up). The implementation we describe here has been included in the atomistic simulator DADOS $[6,8]$. 
As we mentioned above, extended defects can be considered to be neutral as a first approximation. Therefore, our implementation of Fermi-level effects is built on point-defects, and will affect the extended defects only as a consequence. The main tasks that the simulator has to do, related to charged point-defects, are to always maintain the right local ratio between the various charge states (Eq.1) and to include the electric bias effects (Eq.4).

Electrostatic interactions between particles are accounted for through the bias. Additionally, pairing between charges of the same sign is forbidden, reflecting repulsive interaction. The interaction of point-defects with extended defects and with the surface has been enabled only for neutral states. In this way, we get naturally the behavior described by Eq.14.

We calculate $e_{F}$ (and, thus, the carrier concentrations) within the charge neutrality approximation. The translation from discrete charges to charge densities is done using a localdependent averaging radius equal to the Debye-length [22]. If not smoothed distributions are used, incorrectly high diffusivities are obtained, due to overestimation of carrier densities in the neighborhood of dopant atoms.

Substitutional point defect dopants are taken to be always electrically active (ionized). This is a good approximation for shallow dopants (as Boron and Arsenic) even for very high concentrations [7]. In order to account for the high resistivity of highly damaged material, interstitials and vacancies are treated as compensating deep level centers, decreasing the effective value of the dopant concentration.

We have included Fermi-Dirac statistic, but we can also use Maxwell-Boltzmann statistics (non-degeneracy approximation) in order to compare with analytic results. Band-gap narrowing is assessed using Jain and Roulston 's model [23].

\section{SIMULATION EXAMPLES}

The simulation scheme presented above has proven to be efficient for realistic devicedimension process simulations. The inclusion of all the mechanisms mentioned in previous 
sections increases the computation time by less than a factor of 2 with respect to simulations with no charge effects. Typical simulation boxes are of the order of $10^{6} \mathrm{~nm}^{3}$.

Figure 4 shows the good agreement between the differential equation-based model and our atomistic simulations for the case of Arsenic in-diffusion for both intrinsic and extrinsic conditions. The effect of Fermi-level on the pro le shape and depth is evident. The comparison also validate the parameter translation between atomistic and continuum formulation (Eq.11 and equivalent for As).

Figure 5 tests Boron redistribution occurring during post-implant annealing near a $\mathrm{n}^{+} \mathrm{p}$ junction. Boron is depleted from the p side of the junction due to the electric drift, that pushes $\mathrm{B}^{-}$toward the $\mathrm{n}$ side. However, it is interesting to remark that this apparent electric drift was actually associated to a net flux of $\mathrm{B}_{\mathrm{i}}{ }^{0}$, that is neutral. The gradient of $\mathrm{e}_{\mathrm{F}}$ produces a gradient of $\left[\mathrm{B}_{\mathrm{i}}{ }^{0}\right] /\left[\mathrm{B}^{-}\right]$and, in consequence, a diffusion flux of $\mathrm{B}_{\mathrm{i}}{ }^{0}$ toward the n-region that tends to homogenize $\left[\mathrm{B}_{\mathrm{i}}{ }^{0}\right]$. Boron pile-up in the As-rich region is related to the lower value of $\mathrm{D}(\mathrm{B})$ in n-type materials (see Eq.10).

\section{CONCLUSIONS}

We have presented the formalism for the assessment of Fermi-level effects on an atomistic kMC model, as well as a the guidelines for its implementation in an atomistic process simulator. The approach has been illustrated with data analysis and simulation examples for boron and arsenic. The method allows a detailed description of high-doping phenomena situations and is suitable for accurate decanano device process simulation.

This work has been partially supported by the Spanish Government under project BFM 20012250 and by the Castilla y Leon Regional Government under project VA-010/02. 


\section{References}

[1] P. M. Fahey, P. B. Griffin, and J. D. Plummer, Rev. Mod. Phys. 61 (1989) 289.

[2] R. B. Fair, in F. F. Y. Wang (ed.), Impurity Doping Processes in Silicon, NorthHolland, Amsterdam, 1981, p.315.

[3] J. E. Northrup and S. B. Zhang, Phys. Rev. B 47 (1993) 6791.

[4] L. Pelaz, V. C. Venezia, H.-J. Gossmann, G. H. Gilmer, A. T. Fiory, and C. S. Rafferty, Appl. Phys. Lett. 75 (1999) 662.

[5] M. Jaraiz, L. Pelaz, E. Rubio, J. Barbolla, D. J. Eaglesham, H.-J. Gossmann, and J. M. Poate, Mater. Res. Soc. Symp. Proc. 532 (1998) 43.

[6] M. Jaraiz, P. Castrillo, R. Pinacho, I. Martin-Bragado, and J. Barbolla, in D. Tsoukalas and C. Tsamis (eds.), Simulation of Semiconductor Processes and Devices 2001, Springer Verlag, Wien, Austria, 2001, p. 10.

[7] Taurus reference Manual, Synopsys Inc., Mountain View, USA, 2003.

[8] V. Moroz, N. Strecker, and M. Jaraiz, in G. Baccarani, E. Gnani, and M. Rudan (eds.), Proceedings of the $32^{\text {nd }}$ European Solid State Device Research Conference, 2002, p. 299.

[9] C. Rafferty, 1997 International Conference on Simulation of Semiconductor Processes and Devices, IEEE, New York, 1997, p.1.

[10] G. D. Watkins, Mater. Sci. Semicond. Process. 3 (2000) 227.

[11] A. Giese, H. Bracht, N. A. Stolwijk, and D. Baither, Mat. Sci. Eng. B 71 (2000) 160.

[12] J. Zhu, Comp. Mat. Sci. 12 (1998) 309.

[13] H. Bracht, N. A. Stolwijk, and H. Mehrer, Phys. Rev. B 52 (1995) 16542.

[14] H. Bracht, E. E. Haller, and R. Clark-Phelps, Phys. Rev. Lett. 81 (1998) 393.

[15] W. Windl, M. M. Bunea, R. Stumpf, S. T. Dunham, and M. P. Masquelier, Phys. Rev. Lett. 83 (1999) 4345.

[16] G. D. Watkins, Phys. Rev. B 12 (1975) 5824.

[17] D. Mathiot and J. C. Pfister, J. Appl. Phys. 55 (1984) 3518.

[18] R. Pinacho, M. Jaraiz, J. E. Rubio, I. Martin-Bragado, and J. Barbollla, Mat. Sci. Eng. $\mathrm{B}$, in the press.

[19] N. E. B. Cowern, G. F. A. van de Valle, D. J. Gravesteijn, and C. J. Vriezema, Phys. Rev. Lett. 67 (1991) 212.

[20] R. Pinacho, M. Jaraiz, H. J. Gossmann, G. H. Gilmer, J. L. Benton, and P. Werner, Mater. Res. Soc. Symp. Proc. 610 (2001) B7.2. 
[21] N. E. B. Cowern, G. F. A. van de Walle, P. C. Zalm, and D. J. Oostra, Phys. Rev. Lett. 69 (1992) 116.

[22] S. M. Sze, Physics of semiconductor devices, John Willey \& Sons, New York, $2^{\text {nd }}$ ed., 1981.

[23] S. C. Jain and D. J. Roulston, Solid State Electron. 34 (1991) 453. 


\section{Tables}

Table I: Parameters used for charged states of silicon vacancy $\left(\mathrm{V}^{\mathrm{j}}\right)$, silicon self-interstitial $\left(\mathrm{I}^{\mathrm{j}}\right)$ and interstitial boron $\left(\mathrm{B}_{\mathrm{i}}^{\mathrm{j}}\right)$. $\mathrm{D}_{0}$ is the diffusivity prefactor (Eq.3), $\mathrm{E}_{\mathrm{m}}$ the migration energy, and $e_{t}$ the charge level measured from the valence band edge. For $T \neq 0$, we assume that $e_{t}$ scales with band gap energy [22]. Prefactors for equilibrium concentrations and formation energies of $\mathrm{I}^{0}$ and $\mathrm{V}^{0}$ are also shown. Other parameters used in this work but not listed in the Table are $\mathrm{D}_{\mathrm{bk} 0}\left(\mathrm{~B}_{\mathrm{i}}{ }^{-}\right)=5 \mathrm{~cm}^{2} / \mathrm{s}$ and $\mathrm{E}_{\mathrm{b}}\left(\mathrm{B}_{\mathrm{i}}^{-}\right)=0.4 \mathrm{eV} . \mathrm{D}_{\mathrm{bk} 0}$ is the break-up prefactor expressed in diffusivity units $\left(=\left(\lambda^{2} / 6\right) v_{\mathrm{bk} 0}\right) . \mathrm{D}_{\mathrm{bk} 0}\left(\mathrm{~B}_{\mathrm{i}}{ }^{0}\right)$ and $\mathrm{E}_{\mathrm{b}}\left(\mathrm{B}_{\mathrm{i}}{ }^{0}\right)(\mathrm{T})$ can be obtained from Eqs.7 and 8 . We take the jump distance $\lambda$ to be equal to second-neighbor distance $(0.384 \mathrm{~nm})$. In our implementation, the effective capture volume is $\mathrm{v}_{\text {capt }}=3 \lambda^{3}$.

\begin{tabular}{l|ccccccccccc} 
& $\mathrm{V}^{--}$ & $\mathrm{V}^{-}$ & $\mathrm{V}^{0}$ & $\mathrm{~V}^{+}$ & $\mathrm{V}^{++}$ & $\mathrm{I}^{-}$ & $\mathrm{I}^{0}$ & $\mathrm{I}^{+}$ & $\mathrm{B}_{\mathrm{i}}^{-}$ & $\mathrm{B}_{\mathrm{i}}{ }^{0}$ & $\mathrm{~B}_{\mathrm{i}}^{+}$ \\
\hline $\mathrm{D}_{0}\left(\times 10^{3} \mathrm{~cm}^{2} / \mathrm{s}\right)$ & 1 & 1 & 1 & 1 & 1 & 5 & 5 & 5 & 1 & 1 & 1 \\
$\mathrm{E}_{\mathrm{m}}(\mathrm{eV})$ & 0.4 & 0.4 & 0.4 & 0.6 & 0.8 & 1 & 1 & 0.8 & 0.5 & 0.2 & 1.1 \\
$\mathrm{e}_{\mathrm{t}}(\mathrm{T}=0)(\mathrm{eV})$ & 1.06 & 0.6 & & 0.03 & 0.13 & 1 & & 0.2 & 0.72 & & 1.02 \\
{$\left[\mathrm{X}^{0}\right]_{0}^{*}\left(\times 10^{25} \mathrm{~cm}^{-3}\right)$} & & & 1.5 & & & & $10^{3}$ & & & & \\
$\mathrm{E}_{\mathrm{f}}(\mathrm{eV})$ & & & 3.7 & & & & 3.9 & & & &
\end{tabular}




\section{Figure captions}

Fig.1 Formation energies ( $E_{f}$, dashed lines) and diffusion energies ( $E_{\text {dif }}=E_{f}+E_{m}$, solid lines) of charged silicon vacancy $\left(\mathrm{V}^{\mathrm{j}}\right)$ as a function of Fermi-level $\left(\mathrm{e}_{\mathrm{F}}\right)$ using the parameter set of Table $\mathrm{I}$. $\mathrm{e}_{\mathrm{F}}$ is measured from the valence band edge. (Band-gap energy is $E_{g}=1.17 \mathrm{eV}$ for $\left.\mathrm{T}=0 \mathrm{~K}\right)$. Minimum values of $\mathrm{E}_{\mathrm{f}}\left(\mathrm{V}^{\mathrm{j}}\right)$ and $\mathrm{E}_{\mathrm{dif}}\left(\mathrm{V}^{\mathrm{j}}\right)$ are drawn with thick d lines. $E_{\text {dif }}$ int corresponds to the diffusion energy for intrinsic silicon $\left(e_{F}=\right.$ $\left.\mathrm{e}_{\mathrm{i}}\right)$. Open circles indicate the energy crossings corresponding to charge levels $\left(e_{V^{j}}\right)$.

Fig.2 Dashed lines: Energy to form a $B_{i}^{j}$ from a $B^{-}\left(=E_{f}\left(B_{i}^{j}\right)-E_{f}\left(B^{-}\right)\right)$as a function of Fermi-level using the values of Table I. Solid lines: Activation energy of the contribution of $\mathrm{B}_{\mathrm{i}}^{\mathrm{j}}$ for the effective Boron diffusivity in equilibrium conditions, $\mathrm{E}_{\mathrm{dif}}$ (see Eq.9). Migration energies $\left(\mathrm{E}_{\mathrm{m}}\left(\mathrm{B}_{\mathrm{i}}^{\mathrm{j}}\right)\right)$ are the differences between solid and dashed lines. Black dot indicates the value of $\mathrm{E}_{\mathrm{dif}}$ int . The other notation is like in Fig.1.

Fig.3 Arrhenius plot for $B_{i}$ long hop distance $(\Lambda)$. Lines: values calculated using our parameter set for intrinsic, n-doped and p-doped silicon. Symbols: experimental values in quasi-intrinsic conditions $[19,21]$.

Fig.4 Normalized concentration profiles for Arsenic in-diffusion under intrinsic and extrinsic conditions for $800^{\circ} \mathrm{C}$, at different times. Lines: Theoretical diffusion profiles calculated for intrinsic conditions (dashed lines) and for $n=20 n_{i}$ (solid lines) [1]. Symbols: Atomistic kMC simulated profiles, normalized with time, for intrinsic conditions and for $n=20 n_{i}$ (open and filled symbols, respectively). In order to do an even comparison, Maxwell-Boltzmann statistics has been set in our simulation.

Fig.5 Dopant redistribution in a pn junction. Initially boron has an homogeneous concentration close to $2 \times 10^{18} \mathrm{~cm}^{-3}$ (p region). The $\mathrm{n}^{+}$region has been implanted (As, $10 \mathrm{KeV}, 5 \times 10^{14} \mathrm{~cm}^{-2}$ ) and, subsequently, the sample has been annealed by $10 \mathrm{~min}$. at $750^{\circ} \mathrm{C}$. 


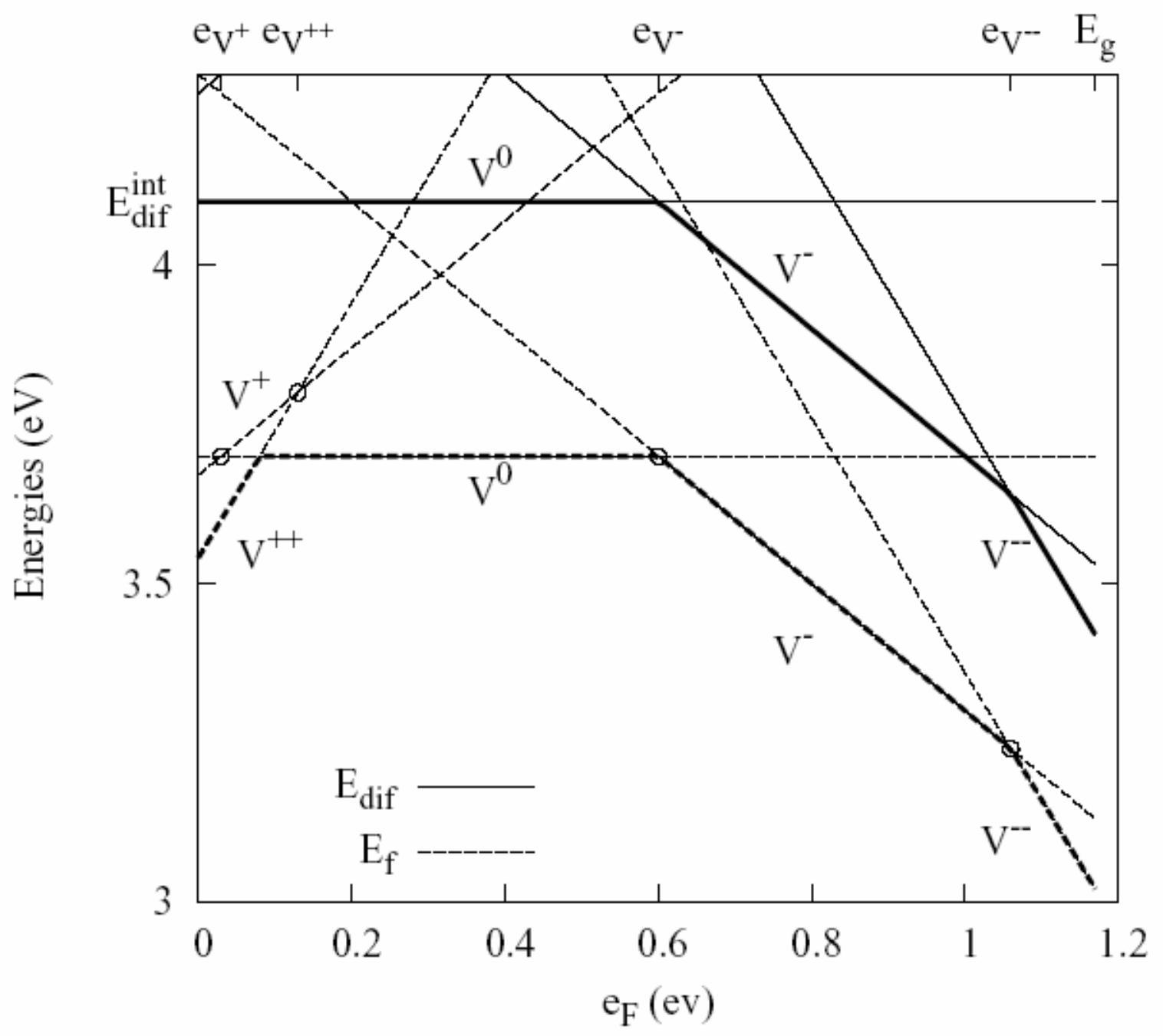

Figure 1 


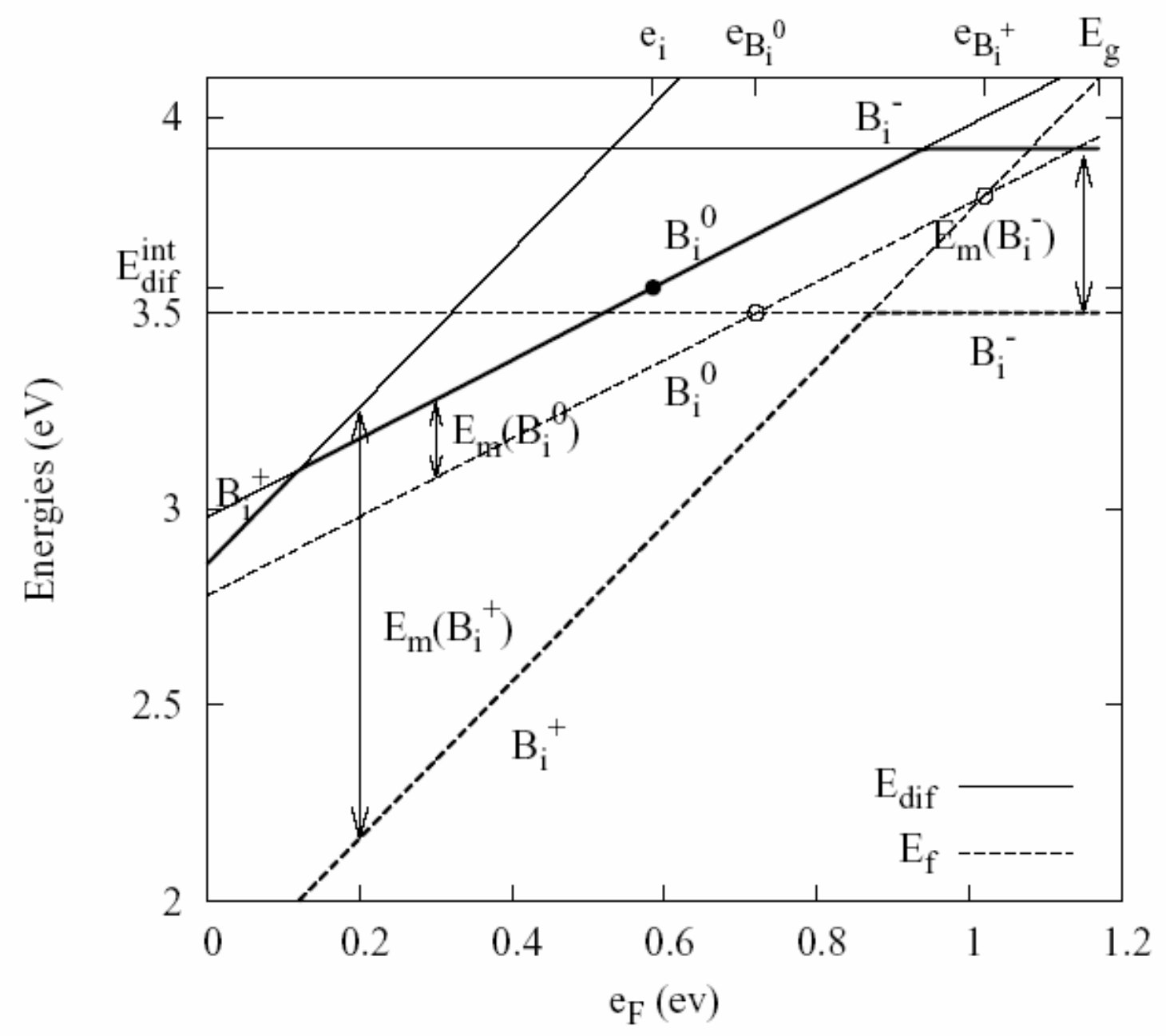

Figure 2 


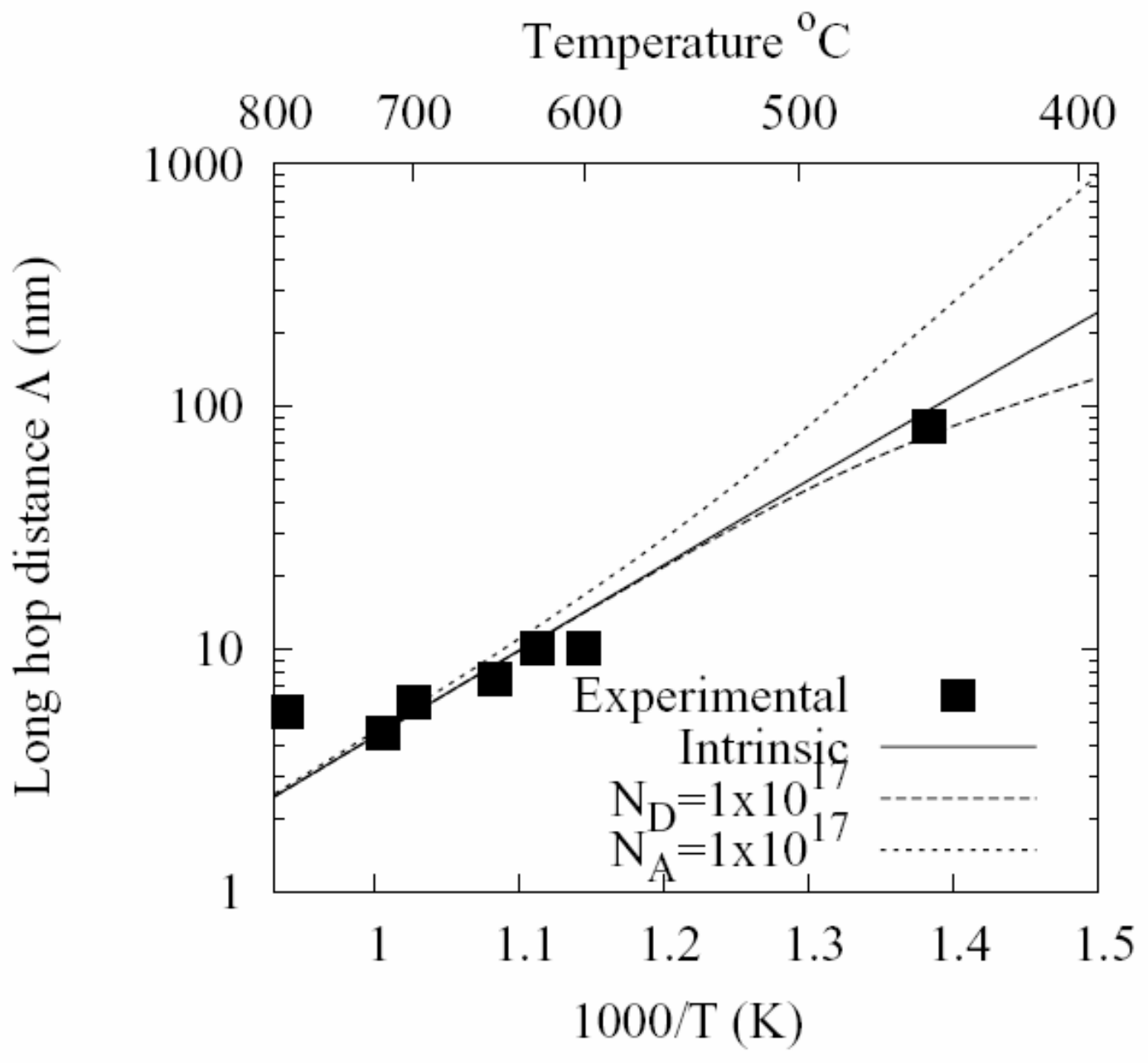

Figure 3 


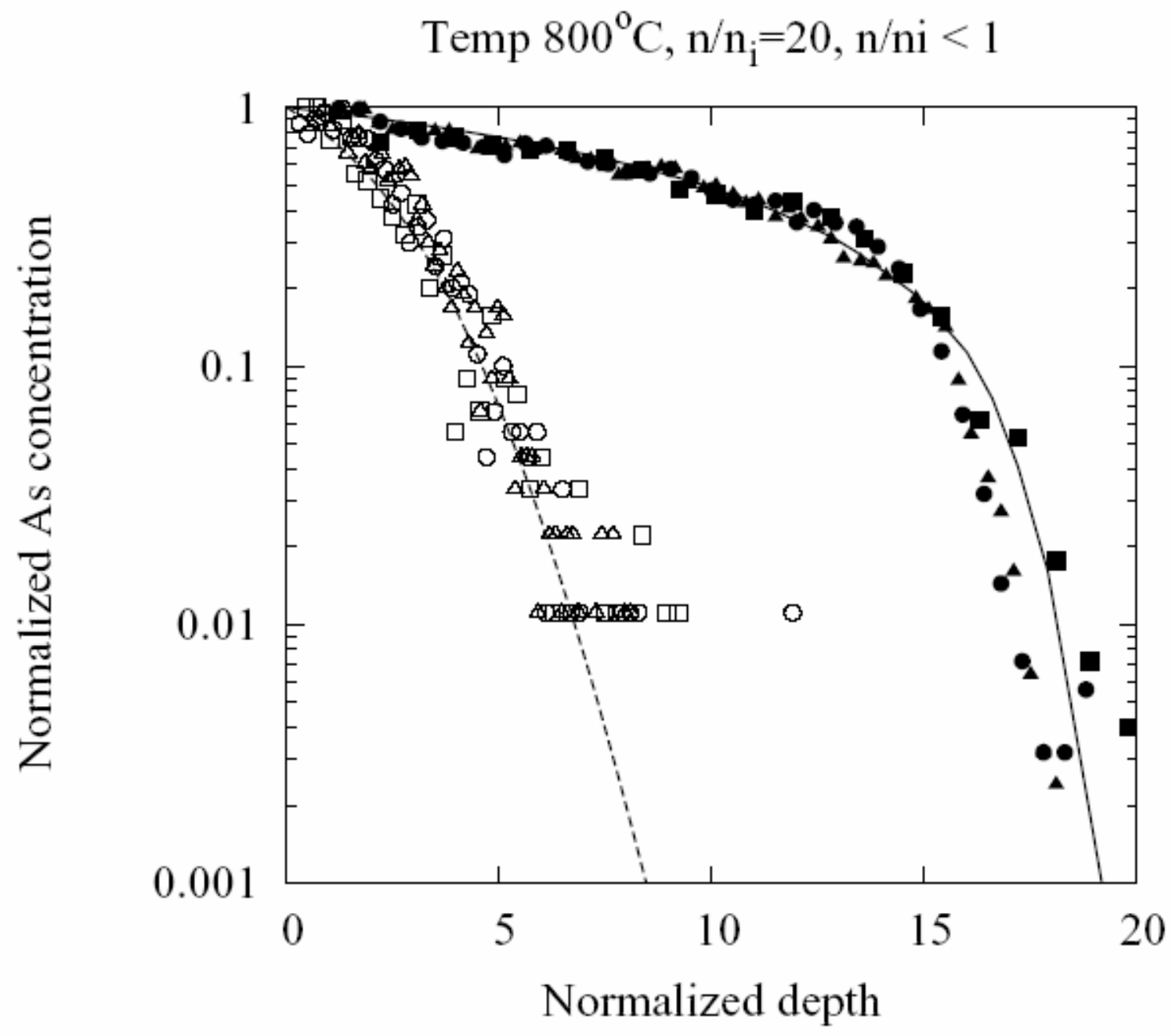

Figure 4 


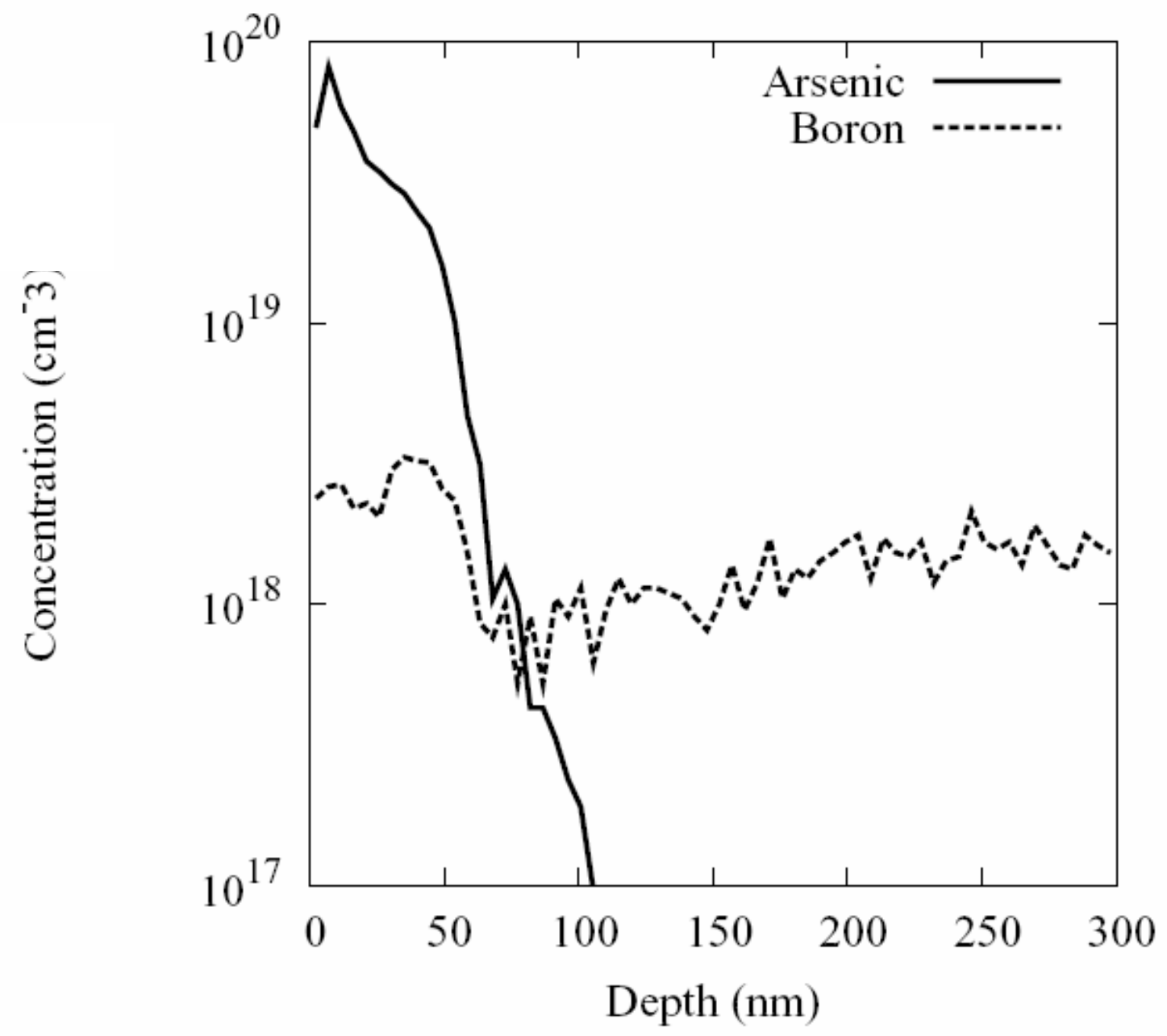

Figure 5 